\title{
New Method for Locker Door with Face Security System Using Backpropagation Algorithm
}

\author{
Y Yusmartato ${ }^{1 *}$, Zulkarnain Lubis ${ }^{2}$, Solly Arza ${ }^{3}$, Zulfadli Pelawi ${ }^{1}$, A Armansah ${ }^{1}$, Abdurrozzaq Hasibuan ${ }^{1}$, Mhd. \\ Asaad $^{4}$
}

${ }^{1}$ Faculty of Engineering, Universitas Islam Sumatera Utara Medan, Indonesia

${ }^{2}$ Electrical of Engineering Institut Teknologi Medan, Indonesia

${ }^{3)}$ Electrical of Engineering Universitas Panca Budi, Indonesia

${ }^{4}$ Faculty of Agriculture, Universitas Islam sumatera Utara Indonesia

*Corresponding author E-mail: yusmartato@ft.uisu.ac.id

\begin{abstract}
Lockers are one of the facilities that people use to store stuff. Artificial neural networks are computational systems where architecture and operations are inspired by the knowledge of biological neurons in the brain, which is one of the artificial representations of the human brain that always tries to stimulate the learning process of the human brain. One of the utilization of artificial neural network is for pattern recognition. The face of a person must be different but sometimes has a shape similar to the face of others, because the facial pattern is a good pattern to try to be recognized by using artificial neural networks. Pattern recognition on artificial neural network can be done by back propagation method. Back propagation method consists of input layer, hidden layer and output layer.
\end{abstract}

Keywords: Locker, Face, Artificial Neural network, Pattern Recognition, Back propagation

\section{Introduction}

Lockers are one of the facilities that people use to store stuff. Lockers commonly found in schools, changing rooms, or facilities for office employees, and so forth. In general, the lockers are equipped with a conventional key lock. However, as technology evolves, the use of conventional keys can be replaced with electric locks in the hope of better security[1], [2].

The conventional identification technique for identifying a person's identity using a password or card is not reliable enough, because the security system can be penetrated, when the password and card are used by unauthorized users[3]. Biometric technology is a technology that uses the human body as an object of detection where biometric technology is used as the basis of detection system of human limbs. In the medical world some members of the human body have differences with other humans [4], [5].

In the description above, hence can be taken a problem statement to be discussed, is: How to apply Back propagation algorithm into the application of locker door security using facial recognition system and develop the system to match the face of the locker owner with a database of stored faces[6], [7].

The objectives of study to be achieved from this paper are: to develop applications that have precision in doing face recognition using Back propagation Algorithm; to produce a Face Recognition Application using Back propagation Algorithm.

The Benefits of Research is utilizing webcam as facial recognition for locker safety. So Gain the application of locker door security with face recognition system using Back propagation Algorithm method. Adding insight, deepening knowledge, and drawing experience in this research so that it can be a useful workforce and ready to face all kinds of challenges in the real world of work.

\section{Literature Review}

Image is a representation (describe), likeness, or imitation of an object. The image is divided into two types. The image that is analogous and images that are digital. Analog image is a continuous image, while digital image is the image that can be processed by computer [8]In general, image processing refers to the processing of 2-dimensional images using a computer.

Artificial neural network (ANN) is a computational system where architecture and operations are inspired by the knowledge of biological neurons in the brain, which is one of the artificial representations of the human brain that always tries to stimulate the learning process of the human brain. Artificial neural networks can be interpreted as information processing systems that have characteristics similar to biological neural networks. Artificial neural networks are created as a generalization of mathematical models of biological neural networks based on the following assumptions.

Artificial neural network (ANN) is computational systems where architecture and operations are inspired by the knowledge of biological neurons in the brain, which is one of the artificial representations of the human brain that always try to stimulate the learning process of the human brain. The neural network can be interpreted as an information processing system that has characteristics similar to that of a biological neural network. Artificial neural networks are created as a generalization of mathematical models of biological neural networks based on the following assumptions [9]:

1. Information processing occurs in a simple element called neuron.

2. The signal is sent between the neurons through the connectors.

3. Connector between neurons has a component/weight that will strengthen or weaken the signal. 
4. To determine the output, each neuron uses an activation function (usually not a linear function) imposed on the number of inputs received. The magnitude of this output is then compared to a threshold.

Back propagation, the artificial neural network weakness consisting of a single screen made the development of artificial neural networks to a halt in the 1970s. The back propagation training algorithm, or some translating it into propagation, was first formulated by Werbos and popularized by Rumelhart with Mc Clelland for use on artificial neural networks. These algorithms include supervised training methods and are designed for operations on artificial multi-layer feed multiples.

Artificial neural networks with a single layer have limitations in pattern recognition. This weakness can be overcome by adding one / several hidden layers between the input and output layers. Although the use of more than one hidden layer has advantages over several cases, but training takes a long time. Thus, generally people start trying with a hidden layer first [10].

\section{Methodology}

Data Collecting Method, is a task or process in the form of analysis and data analysis. In a broad sense as a research design, including the process of planning and conducting research. The steps of preparation in making fire extinguisher robot are as follows:

1. Literature of Study

The author examines references in tins from several scientific papers such as thesis journals and from books.

2. Method of Literature

The literature method, which is collecting data and information by reading references, e-books, websites, documents which include research ever raised, books, articles and journals related to the object of research.

3. Consultation

Conducted in consultation with the supervisor to solve the problems encountered during the manufacture of software and hardware manufacture.

4. Testing Tools

Performed by conducting experiments, testing modules and integrating the module with a program to control the system to be a unified whole and obtained the maximum possible results.

a. System analysis

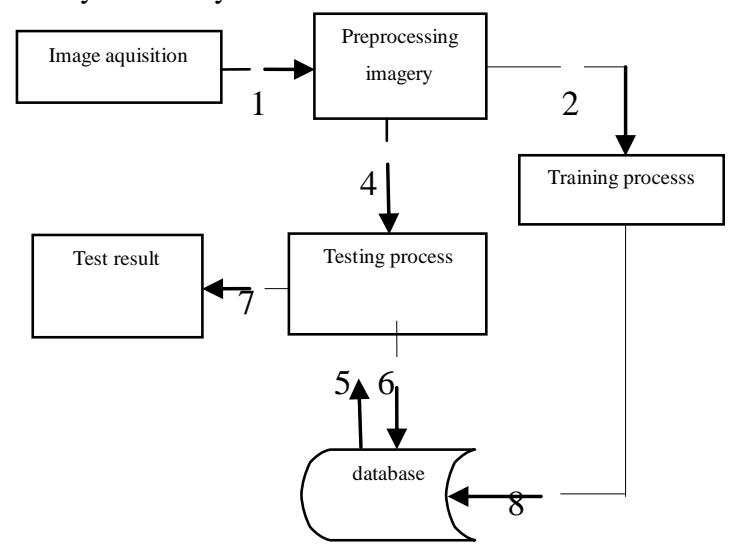

Figure 1: System Analysis Process

b) Analysis of Back propagation Network Architecture

The back propagation network used in this study has an architecture with 24 input units as the data feature used for network input values. Hidden nodes are built using 10 hidden units and the output layer consists of 2 units of output.

The activation function that will be used in the back propagation network built is a bipolar sigmoid activation function. The activation function is used as an activation function on all layers of Artificial Neural Networks either at the time of unit calculation, in the hidden node layer, or in the output layer. The value of the selected learning rate is 0.3 . The use of low learning rate serves to prevent deadlock on the network, where the network stuck to the local minimum.

c) Flowchart system

In Flowchart it can help analytics and programmers to solve problems into smaller segments and help in analyzing other alternatives in operating. In this flowchart will explain how the processes that occur in this system.

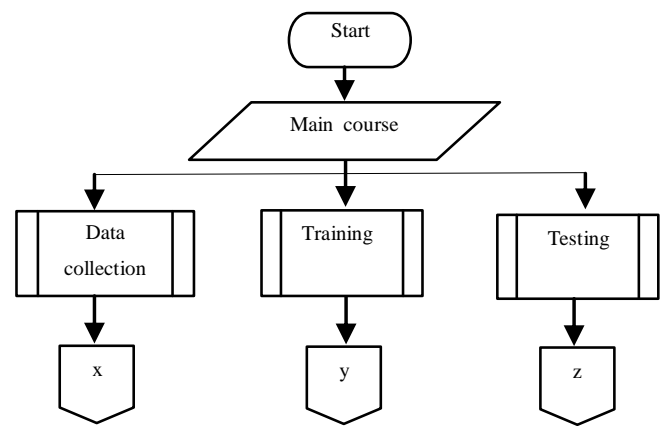

Figure 2: Picture Face Data Flowchart

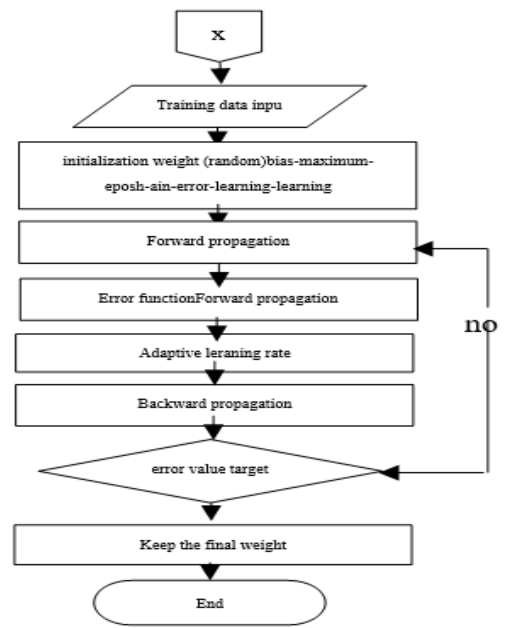

Figure 3: Picture Flowchart Training Back propagation
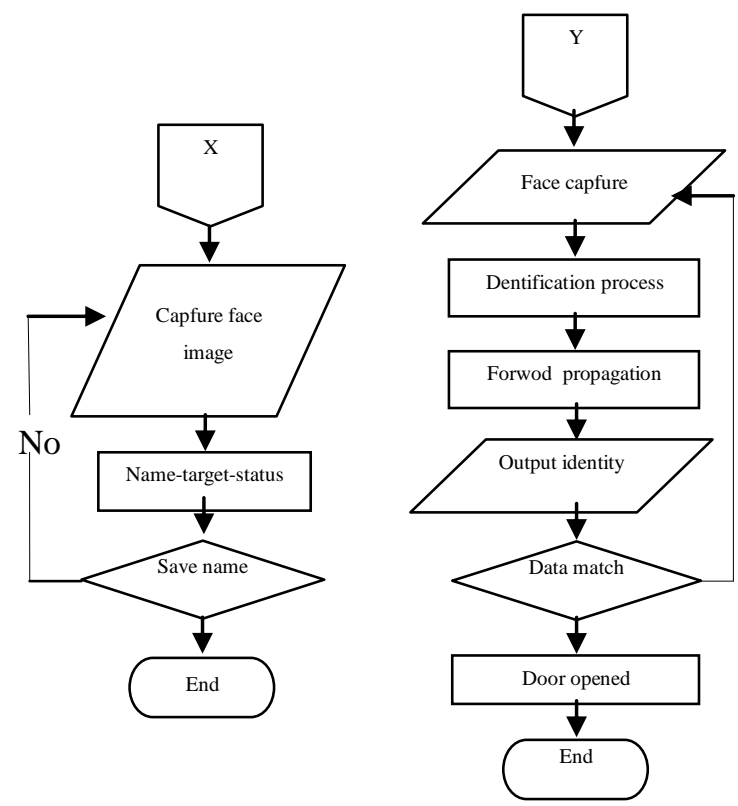

Figure 4: Figure of Flowchart Testing Back propagation 


\section{Results and Discussion}

a. Application Testing

System testing is done to get accurate results. The deficiencies of the system can be detected by means of complex testing then errors can be corrected or eliminated.

b. Main Menu Options

In the main menu there are 4 (four) options are Data Collection, Train, Identify. Themain menuformis a link tootherforms.

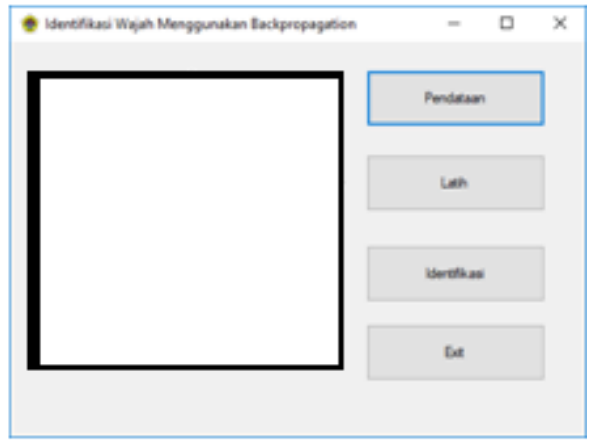

Figure 5: Display Main menu and shows the top view on the application.

c. Options Menu Data Collection

To perform the process of data collection done step:

- Select buttoncapture to take a picture of the face to be saved.

- Then type the name of the face owner in the text box.

- Then type the target in the text box.

- Select the locker ownership status in the combo box.

- Then select the save button.

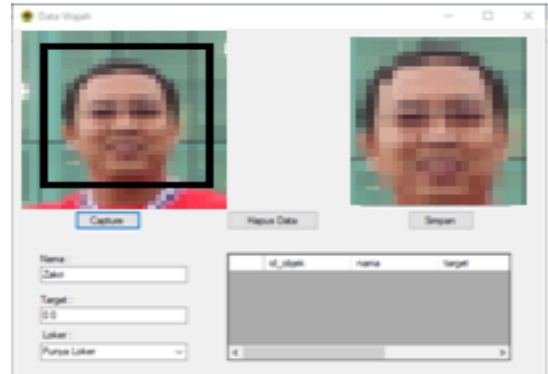

Figure 6: Display the data collection menu

The data collection form serves to enter the data to be used in this research.

d. Choice of Training Menu

To conduct the training process is done step:

- Enter the value of learning rate in combo box.

- Enter the number of iterations (epoch) in the combo box.

- Enter the minimum target error in the combo box.

- Enter the number of hidden nodes in the text box.

- Then select start training button.

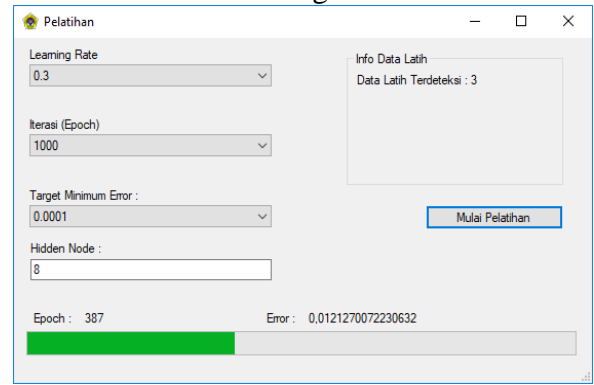

Figure 7: Display of Training Menu

The training form serves to train back propagation nerve tissue. This training process requires several parameters such as the num- ber of iterations, the value of learning rate, the minimum target error value.

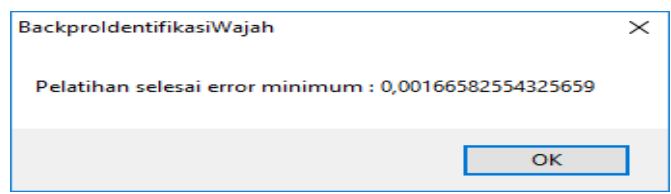

Figure 8: Training Display Completed

Training will be completed if the error value achieved is smaller or equal to the minimum target error value specified.

e. Identify Menu Options

To perform the identification process is done step:

- Select the capture button to take a picture of the face to be identified.

- Then select the identification button.

- The door will open if the output matches the stored database.

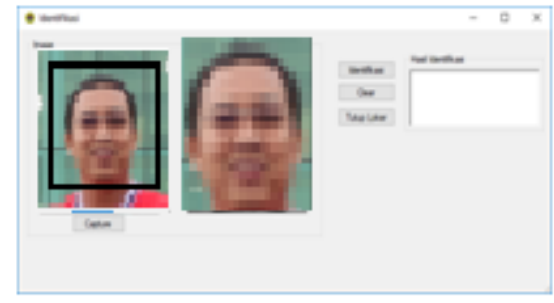

Figure 9: Display Menu Identify

Identification Form serves to perform face identification using facial data that has been captured.

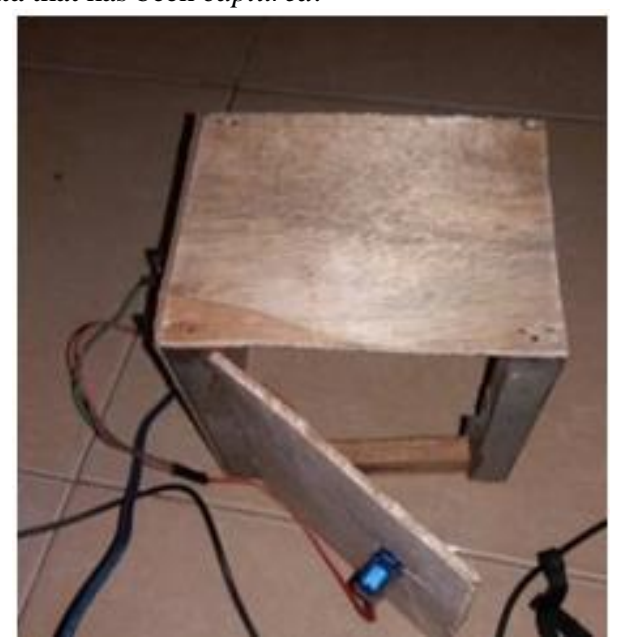

Figure 10. Photo Result of Door Identification Can Be Open

From the results obtained, it can be seen that the facial identification program built can provide good results, where the training and identification process for each face data gives the appropriate results and is able to assist in the security of locker doors.

\section{Conclusion}

Based on the results of trial and discussion of the program conducted, it can be drawn conclusions, namely:

a. Back propagation method can be applied for face recognition.

b. In the face identification process the face position must be fixed therefore during the identification process, the face output results in accordance with the stored face database.

c. The amount of iteration (epoch) affects the length of time required for the training process.

d. Errors in the training process will affect the value of error obtained.

The following are suggestions that the author suggests are expected to further improve the results obtained more: 
a. To develop this application in order to recognize the face in a state of sad expression, happy, angry, and others.

b. For more specific results, one can add a feature extraction process prior to the training and recognition process.

c. To develop this application by adding methods that can provide more accurate results such as fisher face and princypal component analysis methods.

\section{References}

[1] Mulyanto and Eddy, Artificial Intelligence. Yogyakarta: Andi, 2011

[2] O. K. Sulaiman et al., "Bellman Ford algorithm-in RoutingInformation Protocol ( RIP )," vol. 1007, pp. 1-9, 2018.

[3] D. Darmawan, Facial Recognition By Method Backpropagation Using the Camera Infrared CCTV. 2010.

[4] S. J. A. Sihotang, Implementasi Neural Network For Signature Introduction. 2011.

[5] Seprtitahara, Systems Face Recognition (Face Recognition) Using Hidden Method Markov Model (HMM). 2012.

[6] A. Hasibuan, Metodologi Penelitian. 2013.

[7] A. Hasibuan et al., "Performance analysis of Supply Chain Management with Supply Chain Operation reference model," vol. 1007 , pp. 1-8, 2018.

[8] Suyanto, Artifical Intellegence. Bandung, 2014

[9] A. Hermawan, Artificial Neural Network Theory and Application. Yogyakarta: Andi, 2006

[10] J. J. Noon, Network Artificial Neural \& Programming Using Matlab. Yogyakarta: Andi, 2009. 